\title{
How and why should the radiologist look at the placenta?
}

\author{
N. Siauve ${ }^{1,2}$ \\ Received: 4 July 2019 / Accepted: 15 July 2019 / Published online: 7 August 2019 \\ (C) European Society of Radiology 2019
}

\begin{abstract}
This editorial comment refers to the article "Identification of suspicious invasive placentation based on clinical MRI data using textural features and automated machine learning" by Sun et al. in European Radiology.

\section{Key Points}

- Understanding how the placenta works is one of the major challenges facing radiologists.

- New perspectives are opening up for MRI studies of the placenta.

- The authors propose a new approach to placental MRI based on texture analysis and machine learning.
\end{abstract}

Keywords Magnetic resonance imaging $\cdot$ Placenta $\cdot$ Artificial intelligence

\author{
Abbreviations \\ AI Artificial intelligence \\ FGR Fetal growth retardation \\ FIESTA Fast imaging employing steady-state acquisition \\ FISP Fast imaging with steady-state precession \\ HASTE Half-acquisition turbo spin-echo \\ ML Machine learning \\ TA Texture analysis
}

MR imaging is increasingly widely used in modern obstetrics. It is usually performed if the risk of fetal abnormality is high, and it has become an essential tool in fetal medicine. The radiologist plays a major role in detecting birth defects, adjusting clinical management, and establishing the prognosis of affected pregnancies.

By contrast, placental MRI is much less common, and its use is generally limited to that of a complementary problem-solving tool for placental evaluation. However,

This comment refers to the article available at https://doi.org/10.1007/ s00330-019-06372-9.

N. Siauve

nathalie.siauve@aphp.fr

1 Service de Radiologie, Hôpital Louis Mourier, Assistance Publique-Hôpitaux de Paris (APHP), 178, rue des Renouillers, 92701 Colombes Cedex, France

2 INSERM, U970, Paris Cardiovascular Research Center - PARCC, Sorbonne Paris Cité, Paris, France placental abnormalities are of considerable clinical significance, due to their association with high rates of fetal morbidity and mortality. Placenta previa, placental adherence abnormalities, and placental insufficiency are the key defects in this context.

New perspectives are opening up for MRI studies of the placenta and the time is now ripe for systematic and detailed MRI scans of this key organ in pregnancy.

\section{What are the current indications for placental MRI?}

Placental indications for MRI currently concern placental adherence abnormalities (placenta accreta, increta, and percreta). One of the key fundamental issues is the prediction of placental adherence abnormalities in patients with placenta previa. MRI is useful for assessments of placental invasion, particularly in cases of equivocal ultrasound findings or a posteriorly located placenta. MRI can also be used to assess placental invasion, with a mean sensitivity of $90.2 \%$ and specificity of $88.2 \%$ [1].

MRI has recognized added value for the management of placental adherence abnormalities, which requires a multidisciplinary team approach, and it can help to decrease morbidity and mortality. The early detection of placental adherence abnormalities is of crucial importance for determining the most appropriate surgical management technique and preventing hemorrhage during the delivery. 


\section{How is placenta accreta diagnosed?}

The most frequently used sequences for placental evaluation are single-shot fast spin-echo/turbo spin-echo (ssFSE/ssTSE), half-Fourier acquisition single-shot turbo spin-echo (HASTE) T2-weighted, and balanced steady-state free-precession (true FISP or FIESTA). Zaghal et al. described the protocols and techniques involved in his recent review article of MRI evaluation of the placenta [2].

MRI findings for placental adherence abnormalities include dark intraplacental bands on T2-weighted images, disorganized intraplacental vascularity, an abnormal uterine bulge, a heterogeneous placenta, thinning or loss of the retroplacental dark zone on T2-weighted images, myometrial thinning or focal disruption of the myometrium, the invasion of adjacent organs (particularly the bladder), and tenting of the bladder. As early as 2007, Lax et al. described marked placental heterogeneity as an emerging MRI marker of placental invasion, together with irregular thick intraplacental T2 dark bands [3].

\section{What is new?}

Sun et al. propose a new approach to placental MRI based on texture analysis (TA), machine learning (ML), and artificial intelligence (AI). TA provides a quantitative analysis of heterogeneity through the quantification of gray-level patterns and pixel inter-relationships within an image, and the textural features for each image are extracted from several matrices. ML and AI identify the most relevant textural features and can be used to construct an optimal model to facilitate diagnosis.

This approach is particularly suitable for analyses of the placenta, which is known to be heterogeneous. This heterogeneity increases significantly with fetal aging during gestation, through both cotyledon maturation and other aging-associated processes, such as fibrin accumulation and calcification.

Two studies based on TA have recently been published. One of these studies provided the first characterization of the normal placenta as a function of gestational age, based on the textural properties between gestational ages of 23 to 36 weeks [4]. This study confirmed the increase in placental heterogeneity with gestational age. In the other study, Romeo et al. reported their preliminary experiences with a radiomic approach for the diagnosis of invasive placentation in patients with placenta previa, using a combination of ML and TA [5]. They concluded that it was feasible to use this tool to identify the placental tissue abnormalities underlying placental adherence abnormalities.

Sun et al. developed another radiomic analysis pipeline including textural features and automated ML for the prenatal prediction of invasive placentation after 24 weeks of gestational age. It is possible to distinguish abnormal placentation, with its heterogeneous appearance, from the normal maturation on the basis of LoG filtration and TA. A predictive model derived from automated ML that performed well was identified.

Intensity heterogeneity is an emerging MRI marker of placental invasion. However, the reading of the images is subjective and this heterogeneity is difficult for human readers to quantify. TA could be used for quantitative image analysis to assess placental heterogeneity. ML analysis with MRI-derived TA features is a potentially feasible tool for the identification of placental tissue abnormalities underlying placental adherence abnormalities.

\section{Why should the radiologist look at the placenta?}

The placenta plays a crucial role throughout pregnancy, fulfilling a large number of functions. This organ plays a regulatory role extending well beyond nutrition and respiration, also encompassing the endocrine and immune system regulations. As a signaling organ, the placenta produces a myriad of bioactive molecules affecting both maternal and fetal metabolisms and physiologies.

The development and maturation of the placenta during pregnancy is relatively unknown. It is time to harness modern approaches and technologies and to develop new ones to shed light on placental structure, development, and function in real time. A coordinated "Human Placenta Project" has been proposed by Eunice Kennedy Shriver from the NIH National Institute of Child Health and Human Development, with this goal in mind [6].

The combination of TA with MRI meets these objectives fully. Moreover, functional MRI of the placenta is currently being developed, and the promising results obtained to date suggest that it will provide valuable insight into placental function in vivo [7]. T2* and T2 mapping can be used to assess the oxygenation and perfusion of the placenta. Placental oxygenation can also be assessed by the blood-oxygen-level-dependent (BOLD) MRI technique. Several functional MRI techniques are being developed for studies of placental perfusion. Arterial spin-labeling (ASL) MRI quantifies tissue blood flow. Intravoxel incoherent motion (IVIM) MRI quantifies perfusion, the perfusion fraction, and diffusion. Dynamic contrast enhancement (DCE) MRI quantifies tissue blood flow, blood volume fraction, and surface permeability. All these new MRI techniques could help to improve our knowledge of placental structure and function.

\section{What will the future indications for placental MRI be?}

The early detection of placental insufficiency is a major challenge for radiologists. Placental insufficiency is a common 
complication during pregnancy and can lead to severe maternofetal complications, such as fetal growth retardation (FGR), preterm birth, and preeclampsia. These complications are major public health concerns, as FGR is encountered in $5 \%$ of all pregnancies and placental insufficiency is its main cause, and pre-eclampsia occurs in 2 to $6 \%$ of all pregnancies. Placental insufficiency remains difficult to diagnose by MRI or ultrasonography and is often not suspected until late in pregnancy, when complications arise.

The early screening of women with a high risk of developing placental insufficiency by TA may become possible in the near future. Andescavage et al recently described textural changes in vivo in the placenta on MRI, in healthy and high-risk fetuses [8]. Large differences in placental development relating to the onset and severity of FGR and neonatal outcome have been demonstrated in the setting of placental insufficiency.

\section{Conclusion}

Understanding how the placenta works is one of the major challenges facing radiologists, given the crucial role of this organ in fetal development. Major developments are occurring in placental MRI techniques, including the development of TA, ML, and radiomic approaches. Sun et al. provide us an example of the way in which TA could be used to detect invasive placentation. Radiologists need to be aware of this new method for evaluating the placenta, to alert clinicians rapidly in cases of suspected abnormalities, to facilitate appropriate timely management of the mother and the fetus.

Funding The authors state that this work has not received any funding.

\section{Compliance with ethical standards}

Guarantor The scientific guarantor of this publication is Nathalie Siauve.

Conflict of interest The author of this manuscript declares no relationships with any companies, whose products or services may be related to the subject matter of the article.
Statistics and biometry Not applicable because the manuscript is an editorial comment.

Informed consent Informed consent is not required because the manuscript is an editorial comment.

Ethical approval Institutional Review Board approval was not required because the manuscript is an editorial comment.

Methodology

- Not applicable

\section{References}

1. D'Antonio F, Iacovella C, Palacios-Jaraquemada J, Bruno CH, Manzoli L, Bhide A (2014) Prenatal identification of invasive placentation using magnetic resonance imaging: systematic review and meta-analysis. Ultrasound Obstet Gynecol 44:8-16

2. Zaghal AA, Hussain HK, Berjawi GA (2019) MRI evaluation of the placenta from normal variants to abnormalities of implantation and malignancies. J Magn Reson Imaging:1-16

3. Lax A, Prince MR, Mennitt K, Schwebach JR, Budorick NE (2007) The value of specific MRI features in the evaluation of suspected placental invasion. Magn Reson Imaging 25:87-93

4. Do QN, Lewis MA, Madhuranthakam AJ et al (2019) Texture analysis of magnetic resonance images of the human placenta throughout gestation: a feasibility study. PLoS One 14(1):e0211060

5. Romeo V, Ricciardi C, Cuocolo R et al (2019) Machine learning analysis of MRI-derived texture features to predict placenta accreta spectrum in patients with placenta previa. Magn Reson Imaging. https://doi.org/10.1016/j.mri.2019.05.017

6. Guttmacher AE, Maddox YT, Spong CY (2014) The Human Placenta Project: placental structure, development, and function in real time. Placenta 35(5):303-304

7. Siauve N, Chalouhi GE, Deloison B et al (2015) Functional imaging of the human placenta with magnetic resonance. Am J Obstet Gynecol 213(4 Suppl):S103-S114

8. Andescavage N, Dahdouh S, Jacobs M et al (2019) In vivo textural and morphometric analysis of placental development in healthy \& growth-restricted pregnancies using magnetic resonance imaging. Pediatr Res 85(7):974-981

Publisher's note Springer Nature remains neutral with regard to jurisdictional claims in published maps and institutional affiliations. 